\title{
Statistical Signatures of a Targeted Search by Bacteria
}

\author{
Hossein Jashnsaz ${ }^{1}$, Gregory G. Anderson ${ }^{2}$, Steve Pressé ${ }^{3 * \dagger}$ \\ ${ }^{1}$ Department of Physics, Indiana Univ. - Purdue Univ. Indianapolis (IUPUI), Indianapolis, IN 46202 \\ USA \\ 2 Department of Biology, Indiana Univ. - Purdue Univ. Indianapolis (IUPUI), Indianapolis, IN 46202 \\ USA \\ 3 Physics Dept. and School of Molecular Sciences, Arizona State University, Tempe AZ 85287 USA \\ *E-mail: stevenpresse@gmail.com
}

\begin{abstract}
Chemoattractant gradients are rarely well-controlled in nature and recent attention has turned to bacterial chemotaxis toward typical bacterial food sources such as food patches or even bacterial prey. In environments with localized food sources reminiscent of a bacterium's natural habitat, striking phenomena - such as the volcano effect or banding - have been predicted or expected to emerge from chemotactic models. However, in practice, from limited bacterial trajectory data it is difficult to distinguish targeted searches from an untargeted search strategy for food sources. Here we use a theoretical model to identify statistical signatures of a targeted search toward point food sources, such as prey. Our model is constructed on the basis that bacteria use temporal comparisons to bias their random walk, exhibit finite memory and are subject to random (Brownian) motion as well as signaling noise. The advantage with using a stochastic model-based approach is that a stochastic model may be parametrized from individual stochastic bacterial trajectories but may then be used to generate a very large number of simulated trajectories to explore average behaviors obtained from stochastic search strategies. For example, our model predicts that a bacterium's diffusion coefficient increases as it approaches the point source and that, in the presence of multiple sources, bacteria may take substantially longer to locate their first source giving the impression of an untargeted search strategy.
\end{abstract}

Keywords: Chemotaxis, predator-prey, bacteria, point sources, stochastic dynamics, statistical signature

This is the author's manuscript of the article published in final edited form as:

Jashnsaz, H., Anderson, G. G., \& Pressé, S. (2017). Statistical signatures of a targeted search by bacteria. Physical Biology, 14(6), 065002. https://doi.org/10.1088/1478-3975/aa84ea 


\section{Introduction}

Chemotaxis is the process by which bacteria move toward chemoattractant (CA) or recede from chemorepellent (CR) [1-3]. Escherichia coli has served as a model organism establishing basic chemotaxis principles broadly relevant to many bacterial species [3-13]. Such principles include the notion that, rather than steering, bacteria run and re-orient. This dynamical motif, first established for E. coli [4], and subsequently explained on the basis of E. coli's flagellar physiology [14-16], is recapitulated in bacteria equipped with different flagellar physiology [8,17-22]. Adaptation (a bacterium's sensitivity to relative rather than absolute changes in CA) is yet another feature well studied in E. coli [23,24] and observed across other bacterial species [25-28].

Typical bacterial dynamical features, such as adaptation times occurring on second timescales [26] and the fact that bacteria are subject to Brownian motion, give rise to emergent behaviors that are unique to bacteria tracking point sources of CAs. One such example is the recently predicted volcano effect [3,29]. This effect, predicted on the basis of theoretical and computational models, describes the projected shell shape volume that bacteria occupy around rather than on CA patches because of a delay in bacterial response to the detected CA. Thus bacteria run past a source and subsequently re-orient by tumbling thereby spending most of their time around, and not on, the point CA source. In practice, even if the point source of CA is stationary (which is not the case of a bacterial prey), observing such effects would require averaging over multiple bacterial trajectories. For such reasons, we develop a model to predict statistical signatures of a targeted search that is based on known and measurable short-time bacterial dynamics.

Identifying statistical signatures of a targeted search by bacteria toward food patches may be helpful in assessing what behavioral pattern of marine bacteria around food patches may be attributed to chemotactic attraction [6] and may also provide deeper insight on the hunting strategy of bacterial predators toward their prey.

The latter is especially interesting given that it is still debated if the predatory bacterium Bdellovibrio bacteriovorus, $\mathrm{Bb}$, discovered over fifty years ago [30] targets its prey or simply bumps into it at random [9,31-38] despite extensive genetic studies [36, 39-41,41-43] and chemotactic assays [31-33, 35]. For example, the complete genome of Bb HD100 including its methyl-accepting chemotaxis proteins and component-regulatory chemotaxis genes have been identified and fully characterized [41]. These findings 
suggest a small role of chemotaxis in Bb's predation. However, unlike neutrophils chasing bacteria, $\mathrm{Bb}$ are much smaller (less than one micron) and they typically move many times faster than their prey [44] including Salmonella, Pseudomonas, and E. coli [9]. For this reason the motion of Bb neighboring its prey often appears not to specifically target its prey and recent work by our lab suggests that a hydrodynamic mechanism may facilitate Bb's co-localization with its prey [45].

Here we first briefly summarize key features of a statistical model of chemotaxis we've previously developed [46] that incorporates basic known features of chemotaxis. Our focus here is on using our model to make statistical predictions of deviations from an untargeted search neighboring point sources. Our model is not molecular or biochemical in detail. Rather it is a general top-down model applicable to many bacteria that satisfy its basic assumptions (summarized in the section below) and holds equally well for predators seeking prey or foraging bacteria seeking inanimate point sources. 


\section{Methods}

The model. We model the source of $\mathrm{CA}$ as an immobile point food source, located at position $\mathbf{r}_{s}$ relative to some origin, that emits particles (CA molecules detectable by a searcher such as a predator) with rate per unit time $\mathcal{R}$. CA particles diffuse away from the source according to a normal diffusion equation in an infinite domain with open boundary conditions [46,47]. The detection rate (called hit rate), $R\left(\mathbf{r}_{j} \mid \mathbf{r}_{s} ; t\right)$, depends on the distance between $\mathbf{r}_{j}$ and $\mathbf{r}_{s}$, where $\mathbf{r}_{j}$ is the searcher's location and the explicit time dependence accounts for the possibility that the CA source moves in time. CA particles diffuse with coefficient $D$ and decay with some long time constant $\tau$ such that, in the long time limit for a stationary source, the CA profile has reached this steady-state

$$
R\left(\mathbf{r}_{j} \mid \mathbf{r}_{s}\right)=\frac{a \mathcal{R}}{\left|\mathbf{r}_{j}-\mathbf{r}_{s}\right|} \exp \left(-\frac{\left|\mathbf{r}_{j}-\mathbf{r}_{s}\right|}{\lambda}\right)
$$

where $a$ is the searcher's radius, and $\lambda=\sqrt{D \tau}$ is the decay constant for the CA particles.

The number of hits, $h_{j}$, detected by the searcher at position $\mathbf{r}_{j}$ over some time interval $[t, t+\Delta t]$ is then distributed according to a Poisson distribution with spatially varying rate given in Eq. (1)

$$
P\left(h_{j}\right)=\frac{\left(\Delta t R\left(\mathbf{r}_{j} \mid \mathbf{r}_{s}\right)\right)^{h_{j}}}{h_{j} !} \exp \left(-\Delta t R\left(\mathbf{r}_{j} \mid \mathbf{r}_{s}\right)\right)
$$

To model the searcher, we consider the fact that bacteria are typically too small to measure spatial gradients directly. Rather, they perform a temporal comparison of signal [48], integrate signals over the course of seconds $[49,50]$ and use this information to bias their random walk $[23,51,52]$. For this reason, we define a transition probability, $p\left(\mathbf{r}_{j+1} \mid\left\{\mathbf{r}_{i}, h_{i}\right\}_{i \leq j}\right)$, for a bacterium to move to a new position $\mathbf{r}_{j+1}$ depending on (i.e. conditioned on) the bacterium's previous hit history supplied by the conjugate pairs of variables $\left\{\mathbf{r}_{i}, h_{i}\right\}_{i \leq j}$.

In addition, bacteria show adaptation to gradients [24, 25, 27, 28,53-55] (thus, rather than depending explicitly on $h$ or differences in $h, \Delta h$, they depend on $\nabla \log h$ defined below), and are subject to random, Brownian, motion $[56,57]$ as well as internal noise in the chemotaxis signaling cascade $[24,58-60]$ both integrated into a single parameter $\sigma$ [46]. On the basis of these physical observations, we write down the 
following transition probability

$$
p\left(\mathbf{r}_{j+1} \mid\left\{\mathbf{r}_{i}, h_{i}\right\}_{i \leq j}\right)=\mathcal{N} \exp \left(-\frac{\left(\mathbf{r}_{j+1}-\mathbf{r}_{j}-\sum_{i=n}^{n+m} \alpha_{i} \mathbf{f}_{j-i}\right)^{2}}{2 \sigma^{2}}\right) .
$$

The coefficients $\left\{\alpha_{i}\right\}$ describe the weight assigned to information gathered at various time points in the past and have dimensions of length. Furthermore we define

$$
\mathbf{f}_{k} \equiv \frac{\left(\mathbf{r}_{k}-\mathbf{r}_{k-1}\right)}{\left|\mathbf{r}_{k}-\mathbf{r}_{k-1}\right|} \cdot \frac{\left(h_{k}-h_{k-1}\right)}{h_{k}} \equiv \nabla \log h_{k}
$$

where, to be clear, $h_{k}$ are the number of hits at position $\mathbf{r}_{k}$ (i.e. the number of stochastic detections of $\mathrm{CA} / \mathrm{CR}$ molecules by outer membrane chemoreceptors). In addition, the 'memory' $m$ determines how far into the past hit information is considered by the bacterium in selecting its future position while the normalization constant is $\mathcal{N}=\int d \mathbf{r}_{j+1} p\left(\mathbf{r}_{j+1} \mid\left\{\mathbf{r}_{i}, \nabla \log h_{i}\right\}_{i \leq j}\right)$. For convenience, we rewrite $p\left(\mathbf{r}_{j+1} \mid\left\{\mathbf{r}_{i}, h_{i}\right\}_{i \leq j}\right)$ as $p\left(\mathbf{r}_{j+1} \mid\left\{\mathbf{r}_{i}, \nabla \log h_{i}\right\}_{i \leq j}\right)$. Since the bacterium's response only depends on $\nabla \log h$, our model ignores saturation effects that may occur when chemoattractant concentration is very high. When concentrations are high and gradients are steep (i.e. the low fluctuation regime), the searcher in the model closely follows the path of higher CA gradient as would be expected [46]. In implementing Eq. (4), if $h_{j}$ is very small (and could drop to zero) or if sampling a future position is done in a discrete lattice space (where the probability of sampling $r_{j+1}=r_{j}$ is finite), the denominator of Eq. (4) could vanish. We have proposed a way to avoid such complications in a previous paper (Eq. 8 in [46]). However, in all our calculations here, $h_{j}$ is large enough never to be zero, and we sample positions in continuous space where the probability of sampling $r_{j+1}=r_{j}$ is vanishingly small.

We also define an adaptation time, $T_{a}=n \times \Delta t$, which is the time it takes for a bacterium to respond to changes in $\nabla \log h[26]$. Here $\Delta t$ is a data acquisition time (assumed smaller than the adaptation time) and $n$ is an integer to be determined. In our expression for the transition probability (Eq. (3)), an adaptation time $T_{a}$ lasting $n$ time intervals is assumed when we set $\alpha_{i}=0$ for $i<n$. The case where all $\{\alpha\}=0$ coincides with a random 'untargeted' search for prey. In other words, it coincides with a search where no chemical signal information is exploited by the searcher.

While in our previous work [46], our focus was on showing how all unknown parameters, $\{\{\alpha\}, \sigma\} \equiv$ 
$\left\{\left\{\alpha_{0}, \alpha_{1}, \alpha_{2}, \cdots\right\}, \sigma\right\}$, could be directly inferred from single cell tracking data using maximum likelihood methods, here we focus on predictions of our model, valid over broad parameter ranges, assuming parameters have already been learned. In other words, we investigate what statistical signatures of a targeted search emerge under different parameter regimes including searches by bacteria surrounded by multiple sources. These parameters include: the memory length $(m)$, noise $(\sigma)$ and the adaptation time $\left(T_{a}\right)$. Our approach identifies the behaviors one should expect over broad parameter ranges under the assumptions of our model. 


\section{Results}

We first explore the effects arising from the stochasticity of hits on the behavior of searchers and, in doing so, qualitatively illustrate why searchers (such as predators) may appear to adopt an untargeted search strategy when surrounded by multiple prey.

All subfigures in Fig. 1 depict representative trajectories of a single searcher surrounded by food sources located on the radius of a circle $(R=1000 a$ where $a$ is the size of the searcher) centered on the searcher's initial condition. The number of sources neighboring the searcher increases from 1 to 128 . The $3 \mathrm{D}$ trajectories are projected in $2 \mathrm{D}$ for the sake of illustration. As the number of sources around the searcher grow, the searcher initially tumbles more often as it receives conflicting signals from opposing sources. As a result, when surrounded by point sources, somewhat counter-intuitively, the searcher takes longer to locate its first point source; see Fig. 2(A). In fact, the entire distribution of arrival times to the first source broadens substantially and its maximum shifts to longer times as the number of point sources increases (Fig. 2(B)) thereby creating the impression of an untargeted search in an environment crowded by point sources.

What is more, as bacteria approach the source, sudden large variations in the number of hits over length scales comparable to a few bacterial body lengths, $a$, induce erratic changes in the bacterium's behavior - captured in Figs. 3-6 - which we now detail.

First, the volcano effect, previously predicted for bacteria around point sources [3,29], is recapitulated by our model. This effect arises because the bacterium's response time exceeds the time it takes to traverse the immediate neighborhood of a point source. As a result, the bacterium overshoots the source and eventually turns back thus spending most of its time in some shell surrounding the source (Fig. 3(A)). Figure 3(B) shows the bacterial density (normalized over volume) as a function of its radial distance from the source illustrating the distinctive volcano density peak at some distance from the source. While the volcano effect is only in practice observable by considering multiple bacterial trajectories, we can predict the volcano shape given a model parametrized on short trajectories from few bacteria. The same is true for the value of the searcher's diffusion coefficient around a point source that requires less data to characterize.

Intuitively, while one might expect the source to behave as a trap reducing the bacterium's diffusion coefficient as it neighbors the source, the behavior predicted by the model is the precise opposite. The 
bacterium's diffusion coefficient instead peaks dramatically (Fig. 3(C)) as it approaches the source. This is because the gradient gets sharper closer to the source resulting in overall longer steps. See Eqs. (1)-(4).

Figure 3 (D) captures how the searcher may even exhibit noncircular orbits for some time when it gets close enough to the point source for given large memories. This is because for long enough decay times of CA molecules $(\tau)$, Eq. (1) can be approximated as $c(r) \propto 1 / r$ where $r$ is the distance from the source. On the other hand, the chemotactic attraction (force) on the bacterium by the source - which sets up the CA gradient of $c(r)$ in the space - is proportional to the gradient of $\log c(r)$ (see Eq. (1)) generating an approximate central force of $1 / r[61]$.

While we have previously shown howthe entire memory function (set of $\{\alpha\}$ 's) could be inferred from bacterial trajectories (from which the volcano shell density shape could be predicted) [46], it is also possible to do the reverse. That is, it is possible to start from bulk experiments revealing bacterial densities around point sources in order to infer features of bacterial memory. For example, we investigated whether one could deduce the adaptation time from the shape of the density shell arising from the volcano effect (Fig. 3(B)). Figure 4 shows how the density surrounding the source changes as we vary the searcher's precision $(\sigma)$ (Fig. 4(B)) for the (decaying) memory function (set of $\{\alpha\}$ 's) shown in Fig. 4(A). As can be seen from Figs. 4(B), both width and maximum of the density peak are functions of $\sigma$ (and also $n$, data now shown). Broadly we find that, as we increase $\sigma$, the volcano effect disappears. This is because for larger $\sigma$ 's the probability distribution for sampling the next position broadens, resulting in a shallower volcano effect and, ultimately, its elimination altogether. Likewise, we find that as we increase $n$, the peak of the volcano density largely shifts toward larger $r$ 's and it broadens.

Figure 5 quantitatively shows how both width, $W$, and peak location, $R_{0}$, of the density shell - defined in Fig. 5(B) - depend on the adaptation time duration (in unitless $n$ ) and the memory (in unitless $m$ ). The memory function itself used here is shown in Fig. 5(A). In Figs. 5(C) and (D) we show how the volcano shell radii and width vary with $m$ (for a fixed and given adaptation time) while their variation in $n$ for a fixed $m$ is shown in Figs. $5(\mathrm{E})$ and $(\mathrm{F})$. Intuitively, we see that as memory increases, the volcano shell radii $\left(R_{0}\right)$ and width $(W)$ increase because larger memory means more information from the past biases the searcher thereby requiring longer times for the searcher to correct for having overshot the source. This, in turn, results in larger volcano shells (larger $R_{0}$, and $W$ ). However, the response of the volcano shell to adaptation time is more complex. While we see that as $n$ increases, $R_{0}$ broadly increases (particularly for smaller $n$ values), we do not observe significant changes in $W$ with $n$. 
We conclude with a phase diagram, shown in Fig. 6, illustrating the different stationary densities expected from a searcher surrounded by two different sources as a function of the inverse sources distance $(1 / d)$ and the searcher's adaptation time $T_{a}$ (in unitless $n$ ). The phases we recover are shown in Fig. 6 in red and blue. The red region coincides with two volcano shells forming around individual sources. This happens when the distance between both sources is large and adaptation times are shorter. By contrast, the blue region - designating when one volcano shell forms around two sources - arises in the opposite regime. In particular, when adaptation times are long, the searcher's perception of the location of the source is blurred and the searcher perceives two sources as one. 


\section{Discussion}

A targeted search by a bacterium for a point food source is a difficult search problem. Bacteria must at once detect small CA numbers released from the point source and, if the point source moves, bacteria would need to forecast their source's position on the basis of information previously obtained.

Here we have used a statistical top-down chemotaxis model, based on stochastic detection of signal integrated in time by bacteria subject to Brownian motion as well as internal and external signaling noise, to study the dynamics of bacteria around point CA sources. While stochasticity in CA hits received by the searcher gives rise to a search strategy that may naively appear untargeted by eye, our model makes predictions regarding the types of behaviors expected from bacteria targeting a point source even when data is limited. That is, starting from the hypothesis that searchers are drawn toward point sources by CA detection, we identify statistical signatures of a targeted search and show that features of bacterial behaviors, such as adaptation times, may be gleaned from emergent behavior neighboring point sources, such as the radius of a volcano shell.

Our top-down model does not contain signaling pathway-level details. It does however provide a statistical approach appropriate to addressing cell-level dynamical questions as they relate to foraging bacteria. Stochastic detection - a natural consequence of searching for a point source which releases few CAs - may indeed be important in a bacterium's search for CA sources releasing few and thus highly variable number of detectable molecules. Such a search may be particularly relevant to marine bacteria scavenging small patches of food in turbulent ocean waters [5-7,62-64] or predatory bacteria seeking out motile bacterial prey [41]. In both cases, the food patch or the prey could be modeled as a point CA source releasing few CAs. At such low CA concentrations, a model capable of describing chemotaxis in an environment with a variable and noisy number of CA molecules in space is required in order to characterize statistical signatures of targeted search by bacteria. The regime of small number and variable detections by bacteria may be relevant given, for instance, the sensitivity of chemotactic species due to large amplification of signal at the signal transduction pathway [60] and the ultrasensitivity of the flagellar motor to the intracellular concentration of the chemotactic signaling proteins [65]. In other words, bacteria are sensitive and respond to CAs down to a few detection events [60]. For instance, bacterial runs in E. coli can be substantially lengthened (by 30\%) even in nM gradients [60,66].

Our model does have limitations. Beyond immobile CA sources considered thus far, we have not yet 
explicitly considered advection of the fluid in which the searcher seeks a point source or even saturation effects relating to bacteria no longer responding to a point source beyond a critical CA concentration. We have also not yet considered the possibility that prey may detect and respond to approaching predators [67]. In particular, both fluid advection and mobile sources would provide interesting generalizations of our approach as they would result in what would appear as a randomizing influence on the searcher's dynamics that may otherwise again be misinterpreted as an untargeted search.

Indeed, while it is obvious by eye that neutrophils target bacterial prey [68] any determination of a targeted search by bacterial predators for their prey or other bacterial foragers toward a point source is more complex in part because predators, for example, may move faster than their prey [44], are smaller and therefore lack spatial gradient sensing [51,69] and are more susceptible to Brownian forces [70,71] than larger cells. In particular, in the case of predatory-prey bacteria communities, while it is still strongly debated whether the model bacterial predator, Bdellovibrio bacteriovorus [32], actively seeks out its prey [45], a chemotactic search by bacterial predators remains plausible as other bacterial predators such as Myxococcus xanthus are known to seek out their prey chemotactically [72]. 


\section{References}

1. Wadhams G H and Armitage J P 2004 Nature Reviews Molecular Cell Biology 5 1024-1037

2. Eisenbach M 2004 Chemotaxis (Imperial College Press)

3. Bray D, Levin M D and Lipkow K 2007 Curr. Biol. 1712 URL DOI 10.1016/j.cub.2006.11.027

4. Berg H C and Brown D A 1972 Nature 239500

5. Barbara G and Mitchell J 2003 FEMS Microbiol. Ecol. 4479

6. Barbara G M and Mitchell J G 2003 FEMS Microbiol. Ecol. 4399

7. Blackburn N, Fenchel T and Mitchell J 1998 Science 2822254

8. Sockett R and Lambert C 2004 Nat. Rev. Microbiol. 2669

9. Strauch E, Schwudke D and Linscheid M 2007 Future Microbiol. 2 63-73 ISSN 1746-0913 URL http://www.futuremedicine.com/doi/abs/10.2217/17460913.2.1.63

10. Clarke M and Maddera L 2006 Eur. J. Cell Biol. 85 1001-1010

11. Kato J, Kim H E, Takiguchi N, Kuroda A and Ohtake H 2008 J Biosci. Bioeng. 106 1-7

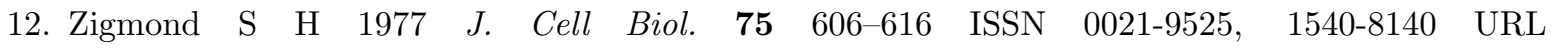
http://jcb.rupress.org/content/75/2/606

13. Astling D P, Lee J Y and Zusman D R 2006 Mol. Microbiol. 59 45-55 ISSN 1365-2958 URL http://onlinelibrary.wiley.com/doi/10.1111/j.1365-2958.2005.04926.x/abstract

14. Turner L, Ryu W S and Berg H C 2000 J. Bacteriol 1822793

15. Berg H C and Anderson R A 1973 Nature 245380

16. Boehm A, Kaiser M, Li H, Spangler C, Kasper C A, Ackermann M, Kaever V, Sourjik V, Roth V and Jenal U 2010 Cell 141 107-116 ISSN 0092-8674 URL http://www.sciencedirect.com/science/article/pii/S009286741000019X

17. Xie L, Altindal T, Chattopadhyay S and Wu X L 2011 Proc. Natl. Acad. Sci. USA 108 2246-2251 ISSN 0027-8424, 1091-6490 URL http://www.pnas.org/content/108/6/2246 
18. Son K, Guasto J S and Stocker R 2013 Nature Phys. 9 494-498 ISSN 1745-2473 URL http://www.nature.com/nphys/journal/v9/n8/abs/nphys2676.html

19. Cai Q, Li Z, Ouyang Q, Luo C and Gordon V D 2016 mBio 7 e00013-16 ISSN , 2150-7511 URL http://mbio.asm.org/content/7/2/e00013-16

20. Morse M, Colin R, Wilson L and Tang J 2016 Biophys. J. 110 2076-2084 ISSN 0006-3495 URL http://www.sciencedirect.com/science/article/pii/S0006349516301096

21. Hill K 2003 Euk. Cell 2200

22. Herzog B and Wirth B 2012 Appl. Environ. Microbiol. 781670

23. Tu Y 2013 Annu. Rev. Biophys. 42337

24. Kalinin Y V, Jiang L, Tu Y and Wu M 2009 Biophys. J. 962439

25. Barkai N and Leibler S 1997 Nature 387913

26. U Alon M G Surette N B and Leibler S 1999 Nature 397168

27. Spiro P, Parkinson J S and Othmer H 1997 Proc. Natl. Acad. Sci. USA 147263

28. Goryanin I I and Goryachev A B 2011 Advances in Systems Biology (Springer)

29. Simons J E and Milewski P A 2010 Math. Comput. Model. 531374

30. Stolp H and Starr M P 1963 Antonie van Leeuwenhoek 29 217-248 ISSN 0003-6072, 1572-9699 URL http://link.springer.com/article/10.1007/BF02046064

31. Straley S C and Conti S F 1974 J. Bacteriol. 120 549-551 ISSN 0021-9193 URL http://www.ncbi.nlm.nih.gov/pmc/articles/PMC245800/

32. Straley S C and Conti S F 1977 J. Bacteriol. 132 628-640 ISSN 0021-9193, 1098-5530 URL http://jb.asm.org/content/132/2/628

33. LaMarre A G, Straley S C and Conti S F 1977 J. Bacteriol. 131 201-207 ISSN 0021-9193, 1098-5530 URL http://jb.asm.org/content/131/1/201 
34. Varon M and Zeigler B P 1978 Appl. Environ. Microbiol. 36 11-17 ISSN 0099-2240, 1098-5336 URL http://aem.asm.org/content/36/1/11

35. Straley S C, LaMarre A G, Lawrence L J and Conti S F 1979 J. Bacteriol. 140 634-642 ISSN 0021-9193, 1098-5530 URL http://jb.asm.org/content/140/2/634

36. Lambert C, Smith M C M and Sockett R E 2003 Environ. Microbiol. 5 127-132 ISSN 1462-2920 URL http://onlinelibrary.wiley.com/doi/10.1046/j.1462-2920.2003.00385.x/abstract

37. Lambert C, Morehouse K A, Chang C $\quad$ Y and Sockett $\mathrm{R}$ E 2006 Curr. Opin. Microbiol. 9 639-644 ISSN 1369-5274 URL http://www.sciencedirect.com/science/article/pii/S1369527406001548

38. Lambert C, Hobley L, Chang C Y, Fenton A, Capeness M and Sockett L 2008 A Predatory Patchwork: Membrane and Surface Structures of Bdellovibrio bacteriovorus Advances in Microbial Physiology vol 54 ed Poole R K (Academic Press) pp 313-361 URL http://www.sciencedirect.com/science/article/pii/S0065291108000052

39. Cotter T and Thomashow M 1992 Journal of bacteriology 174 6011-6017

40. Lambert C, Morehouse K A, Chang C Y and Sockett R E 2006 Current opinion in microbiology 9 $639-644$

41. Rendulic S, Jagtap P, Rosinus A, Eppinger M, Baar C, Lanz C, Keller H, Lambert C, Evans K J, Goesmann A, Meyer F, Sockett R E and Schuster S C 2004 Science 303 689-692 ISSN 0036-8075, 1095-9203 URL http://www.sciencemag.org/content/303/5658/689

42. Hobley L, Fung R K, Lambert C, Harris M A, Dabhi J M, King S S, Basford S M, Uchida K, Till R, Ahmad R et al. 2012 PLoS Pathog 8 e1002493

43. Rotem O, Nesper J, Borovok I, Gorovits R, Kolot M, Pasternak Z, Shin I, Glatter T, Pietrokovski S, Jenal U et al. 2016 Journal of bacteriology 198 127-137

44. Lambert C, Evans K J, Till R, Hobley L, Capeness M, Rendulic S, Schuster S C, Aizawa S I and Sockett R E 2006 Mol. Microbiol. 60 274-286 ISSN 1365-2958 URL http://onlinelibrary.wiley.com/doi/10.1111/j.1365-2958.2006.05081.x/abstract 
45. et al J Biophysical Journal 112 ISSN XX URL XX

46. Jashnsaz H, Nguyen T, Petrache H I and Pressé S 2015 PLoS ONE 10 e0140428

47. Vergassola M, Villermaux E and Shraiman B I 2007 Nature 445406

48. Segall J E, Block S M and Berg H C 1986 Proc. Natl. Acad. Sci. USA 838987

49. BROWN D A and BERG H C 1974 Proc. Natl. Acad. Sci. USA 711388

50. Berg H and Tedesco P 1975 Proc. Natl. Acad. Sci. USA 723235

51. Macnab R M and Koshland D E 1972 Proceedings of the National Academy of Sciences $692509-$ 2512 ISSN 0027-8424, 1091-6490 URL http://www.pnas.org/content/69/9/2509

52. Berg H C and Purcell E M 1977 Biophys. J. 20193

53. Jiang L, Ouyang Q and Tu Y 2010 PLoS Comput. Biol. 6 e1000735

54. Hauri D C and Ross J 1995 Biophysical journal 68 708-722

55. Segel L A, Goldbeter A, Devreotes P N and Knox B E 1986 Journal of theoretical biology 120 $151-179$

56. Berg H C 1993 Random Walks in Biology (Princeton University Press)

57. Patnaik P R 2012 Bioscience 621030

58. Bren A and Eisenbach M 2000 Journal of bacteriology $1826865-6873$

59. Park H, Oikonomou P, Guet C C and Cluzel P 2011 Biophysical journal 101 2336-2340

60. Sourjik V and Berg H C 2010 Proc. Natl. Acad. Sci. USA 99123

61. Tsori $\mathrm{Y}$ and Gennes P G d 2004 Europhys. Lett. 66 599 ISSN 0295-5075 URL http://iopscience.iop.org/0295-5075/66/4/599

62. Seymour J R, Marcos, and Stocker R 2009 Am. Nat. 173 pp. E15-E29 ISSN 00030147 URL http://www.jstor.org/stable/10.1086/593004

63. Azam F and Malfatti F 2007 Nature Rev. Microbiol. 5 782-791 ISSN 1740-1526 URL http://www. nature.com/nrmicro/journal/v5/n10/abs/nrmicro1747.html 
64. Stocker R 2012 Science $338 \quad 628-633$ ISSN $0036-8075, \quad 1095-9203 \quad$ URL http://www.sciencemag.org/content/338/6107/628

65. Cluzel P, Surette M and Leibler S 2000 Science 287 1652-1655

66. Sourjik V 2004 Trends Microbiol. 12569

67. Sengupta A, Kruppa T and Lwen $\mathrm{H} \quad 2011$ Phys. Rev. $\quad$ E $\quad \mathbf{8 3} 031914$ URL http://link.aps.org/doi/10.1103/PhysRevE.83.031914

68. Shen L, Smith J M, Shen Z, Hussey S B, Wira C R and Fanger M W 2006 Immunology 117 205-212 ISSN 0019-2805 URL http://www.ncbi.nlm.nih.gov/pmc/articles/PMC1782216/

69. Berg H C and Purcell E M 1977 Biophysical Journal 20 193-219 ISSN 0006-3495 URL http://www.ncbi.nlm.nih.gov/pmc/articles/PMC1473391/

70. Berg H C 2004 E. coli in motion (Springer)

71. Sokolov A, Apodaca M M, Grzybowski B A and Aranson I S 2010 Proceedings of the National Academy of Sciences 107 969-974 ISSN 0027-8424, 1091-6490 URL http://www.pnas.org/content/107/3/969

72. Berleman J E, Scott J, Chumley T and Kirby J R 2008 Proc. Natl. Acad. Sci. USA 105 17127-17132 ISSN 0027-8424, 1091-6490 URL http://www.pnas.org/content/105/44/17127 


\section{Figure Legends}

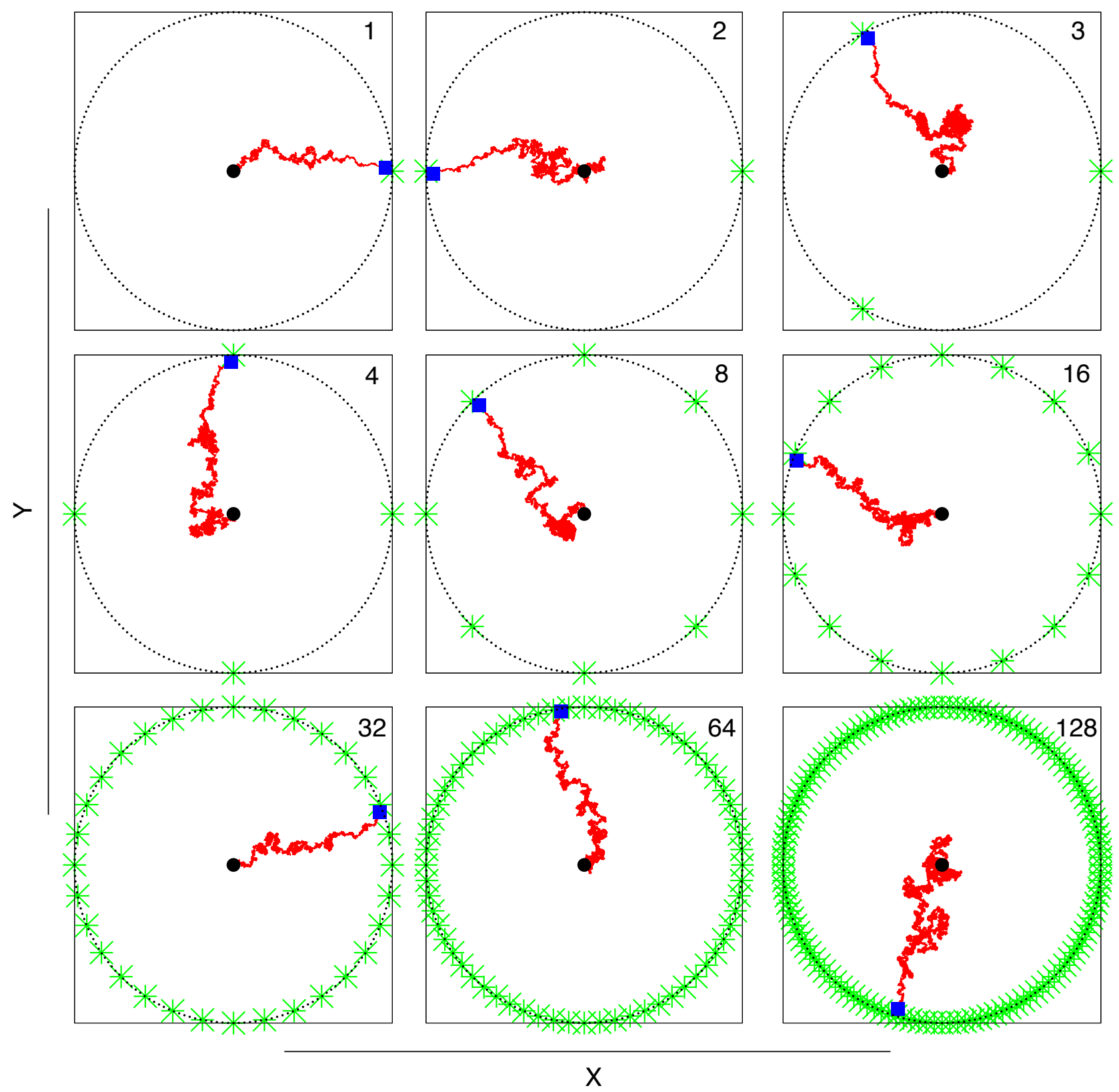

Figure 1. Bacterial search strategies may appear untargeted if searchers are surrounded by multiple CA sources. All point sources have identical emission properties and were placed in the $z=0$ plane equidistantly from the initial location of the mobile searcher (placed at the origin, black circle). The number of point sources surrounding the searcher are $1,2,3,4,8,16,32,64$, and 128 . The green stars denote the locations of the point sources on a circle of radius $R$. To generate all trajectories, the next step is allowed to be taken until the distance between the searcher and the source is less than $50 a$ (trajectory ends are denoted by blue squares). We used $\alpha_{0} / a=10, \sigma / a=1, R / a=1000, n=0$, and $m=10$ (all $\alpha_{i}$ are the same as $\alpha_{0}$ and zero beyond the given memory). Simulation is done in 3D, but a $2 \mathrm{D}$ projection in the $z=0$ plane is shown. For all simulations throughout this paper we used $\Delta t=0.1 s$, $a=1 \mu \mathrm{m}, \mathcal{R}=1.7 \times 10^{7} \mathrm{~s}^{-1}$, and $\lambda=10^{5} \mu \mathrm{m}$. 

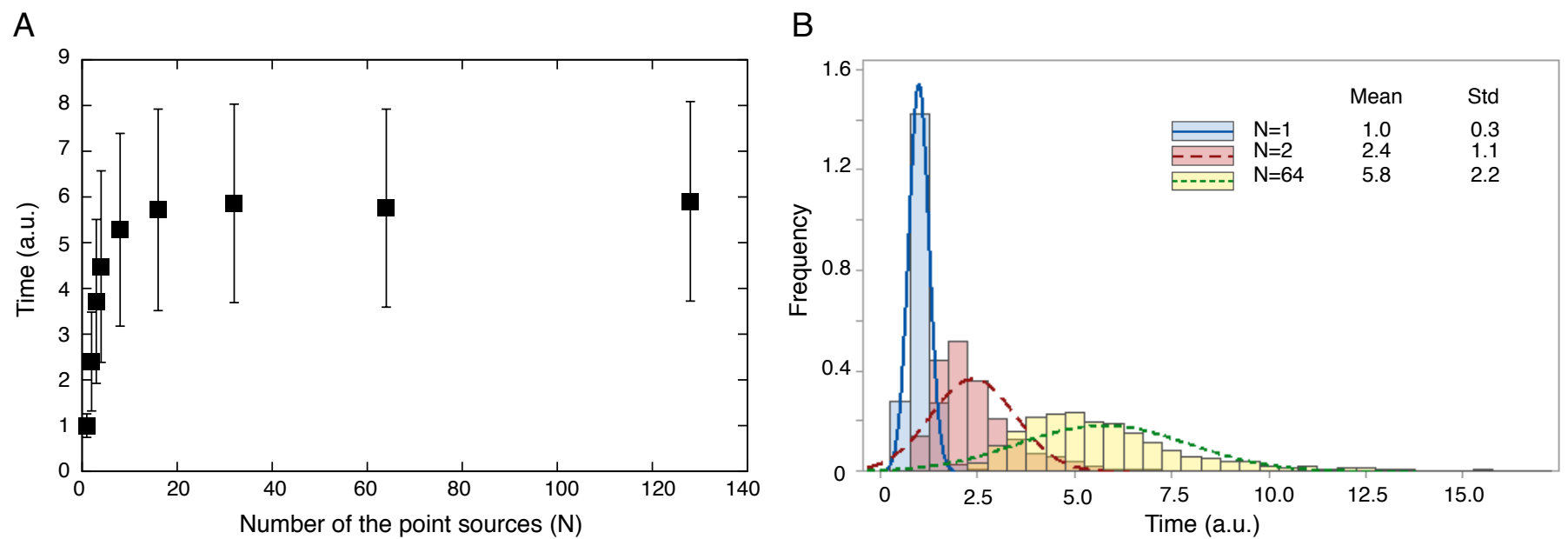

Figure 2. It takes longer for the searcher to locate the first source as the number of sources increases. (A) The mean time taken for a bacterium to find the first point source increases with the number of point sources surrounding the searcher. Each data point represents the average from 2000 simulations with its associated standard deviation shown as error bars. In (B) we show histograms (drawn from 2000 simulations) of the time taken by bacteria to find the first point source for 1,2 or 64 sources surrounding the searcher. As the number of sources increases, the histograms become broader and shift to the right. The dashed lines are normal fits to the corresponding histograms with means and standard deviations (Std) provided in the inset. Time in both are unitless (they are reported with respect to mean time for $N=1$ ). The trajectories are stopped when the searcher gets to a distance of $50 a$ from the first source. We used $\alpha_{0} / a=10, \sigma / a=1, R / a=1000, n=0$, and $m=10$. 

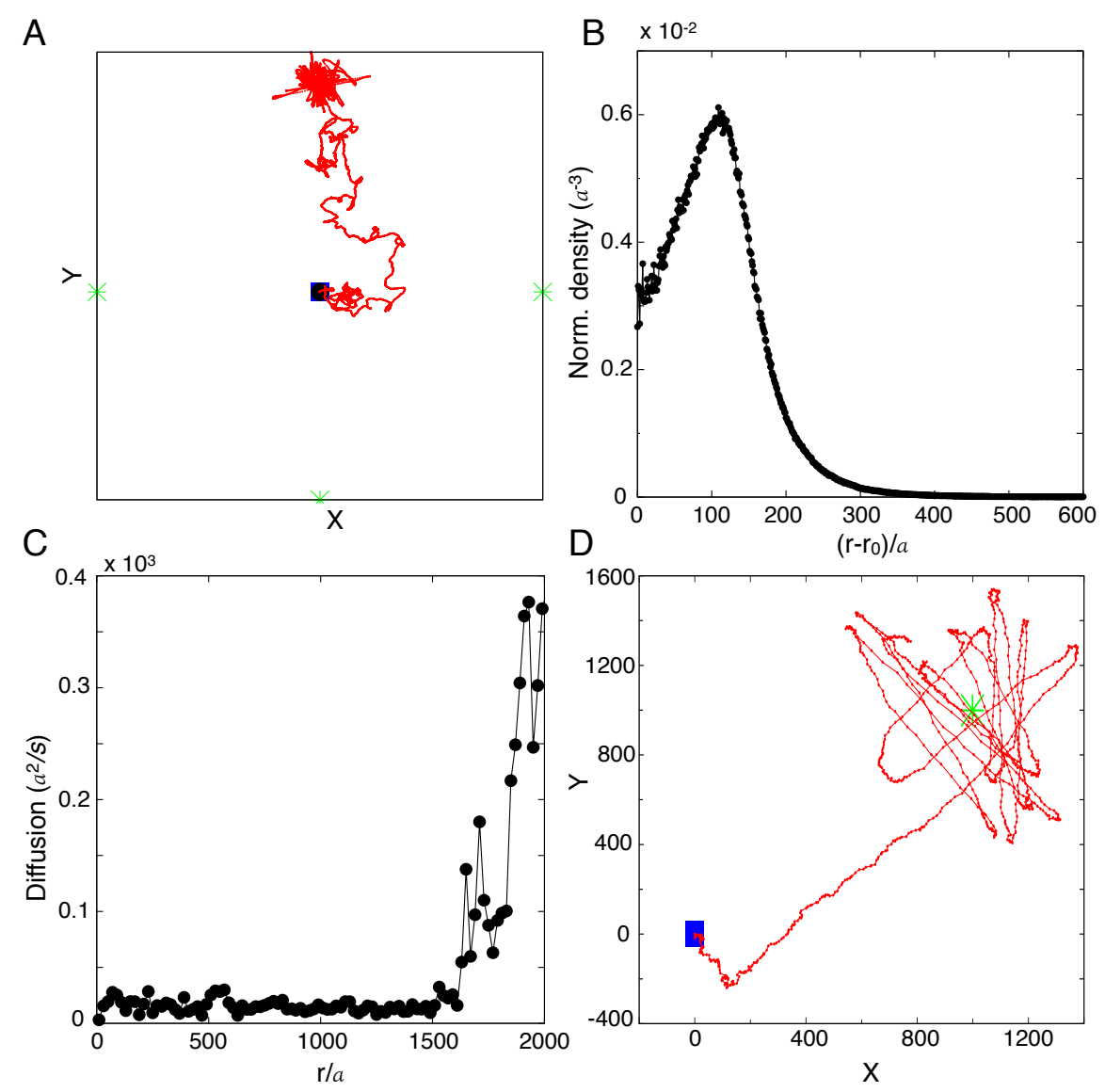

Figure 3. Trajectories, the volcano effect and diffusion coefficients shown for a configuration with 4 point sources. (A) A trajectory and (B) its coinciding normalized density distribution (generated using 100 trajectories) illustrating the volcano effect with $r_{0}$ designating the position of the first point source located. (C) The diffusion coefficient of the searcher increases as the searcher approaches a point source (at 2000 on the $\mathrm{x}$-axis) with $r$ the radial distance from the starting point (the center of the circle shown in $(\mathrm{A})$ ). We calculate the diffusion coefficient from simulated trajectory data, more specifically, we calculate mean square displacements over each step. In (A)-(C) trajectories are run for 20,000 steps. We used $\alpha_{0} / a=30, \sigma / a=1, R / a=2000, n=0$, and $m=30$. (D) The searcher appears to make loops as it approaches the point source. In other words, it runs past the source, tumbles over some distance and then runs past the source once more. This type of trajectory - which arises typically for larger $m$ and larger $\sigma$ because these give rise to broader turns and larger loops - was generated using 1000 steps, starting from $(0,0,0)$, with a point source located at $(1000,1000,1000)$. Here we used $\alpha_{0} / a=30, \sigma / a=5, n=5$, and $m=50$. The simulation is done in 3D but a projected 2D plot of the results in the $z=0$ plane is shown for all trajectories used in this figure. 

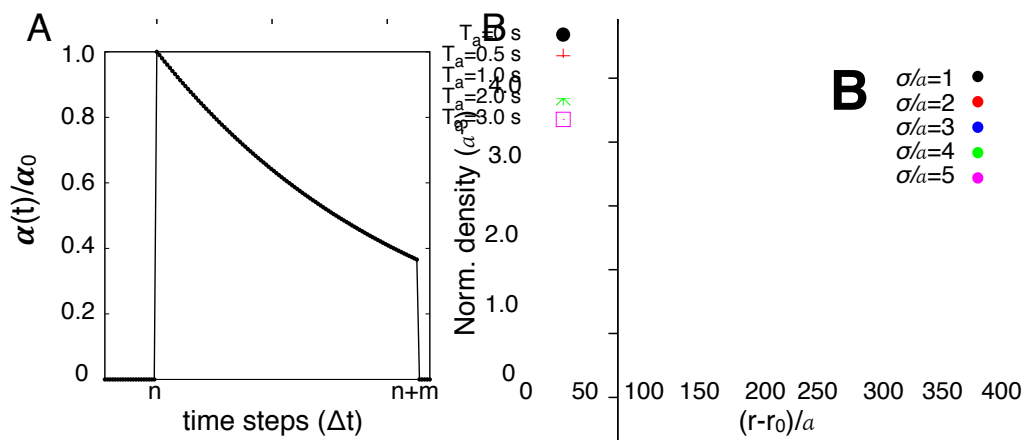

Figure 4. The volcano ring's shape around point sources depends on the precision $(\sigma)$. (A) Here we show the decaying memory coefficients that we used for the results presented in this figure. In previous work, we showed how to infer these model parameters (see Fig. 5 in [46] for the case of a decaying memory). Here we investigate the effects of precision $(\sigma)$ on the volcano shape for such decaying memory. Specifically, we used $\alpha(t) / \alpha_{0}=(0.9)^{t-T_{a}}$ for $T_{a}<t<T_{a}+10(n<i<n+100)$ and 0 elsewhere with $\alpha_{0} / a=30$. (B) Here we show the volcano density profile for different value of $\sigma$. We used an adaptation time of $n=5$ and the plot is obtained from an average of 200 trajectories simulated in 3D. 

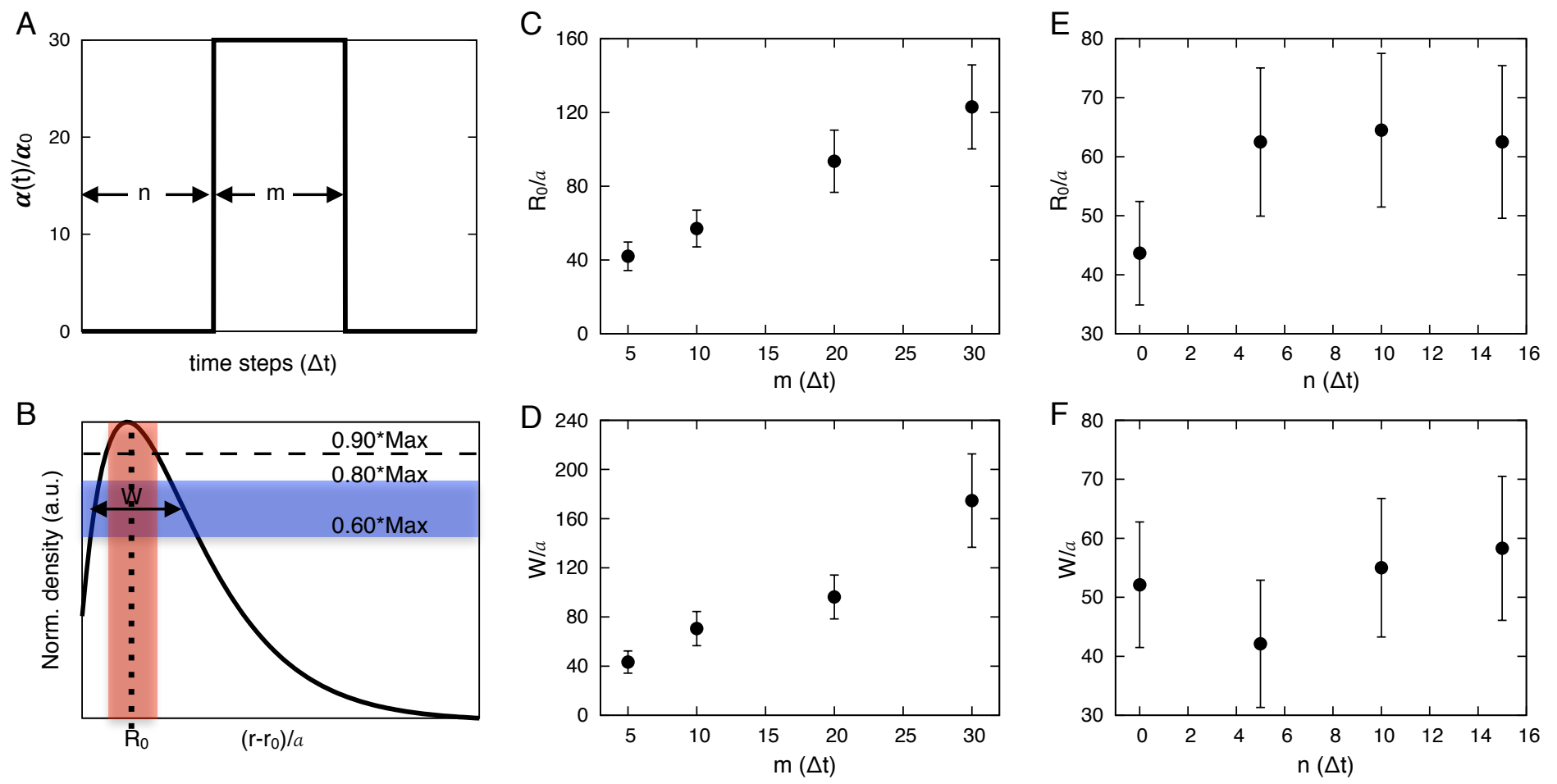

Figure 5. The peak location $\left(R_{0}\right)$ and width $(W)$ of the volcano ring changes with the adaptation time $(n)$ and the memory $(m)$. (A) A cartoon showing how the functional form for $\alpha(t)$ depends on $n$ and $m$. Here we use a finite constant memory with a finite delay (a square profile). We change $n$ and $m$ to quantitatively investigate how the volcano changes versus memory and the adaptation time. (B) An illustration of the quantities $R_{0}$ and $W . R_{0}$ is the peak location (the radius) and $W$ is the width of the volcano shell with $R_{0}$ defined as the average of all radii with densities higher than $90 \%$ of the maximum density, and $W$ defined as the average width of the volcano with densities between $60 \%$ and $80 \%$ of the maximum density. In other words, $R_{0}$ is the average radius of the density profile falling within the red area, and $W$ is the average width of the density falling within the blue area. (C) $R_{0}$ vs $m$ for fixed $n=5$. (D) $W$ vs $m$ for fixed $n=5$. (E) $R_{0}$ vs $n$ for fixed $m=10$. (F) $W$ vs $n$ for fixed $m=10$. These data points are obtained from 100 independent trajectories in (C) and (D), and 500 independent trajectories in (E) and (F), all simulated in 3D. Error bars in all plots reflect a single standard deviation. A square-memory function was used here in order to allow us to vary $n$ and $m$ independently without introducing additional parameters describing the memory's decay. 
Figure 6. A phase diagram for the volcano shell formed around two sources showing its dependence on the inverse distance between two sources $(1 / d)$ and the adaptation time $\left(T_{a}=n \times \Delta t\right)$. The red region coincides to the parameter regime where two volcano shells form around individual sources, while the blue region is for a unique volcano shell (insets are for illustration). To distinguish the single-shell region (blue) from the two-volcano-shells region (red), we use the Spearman's rank correlation coefficient and seek non-monoticity of the radial bacterial density as we move away from a first source defined as the origin. That is, if a second peak is statistically significant (which happens for the case of two distinct volcano shells), it will be detected as a violation of the expectation that the profile beyond the first peak should decay monotonically. More precisely, absolute values for this coefficient larger than 0.99 correspond to the blue region, i.e. the phase with only one volcano shell surrounding both sources. Data from 200 trajectories simulated in 3D was collected to calculate each density profile. We used the memory function given in Fig. 4(A) for these results.

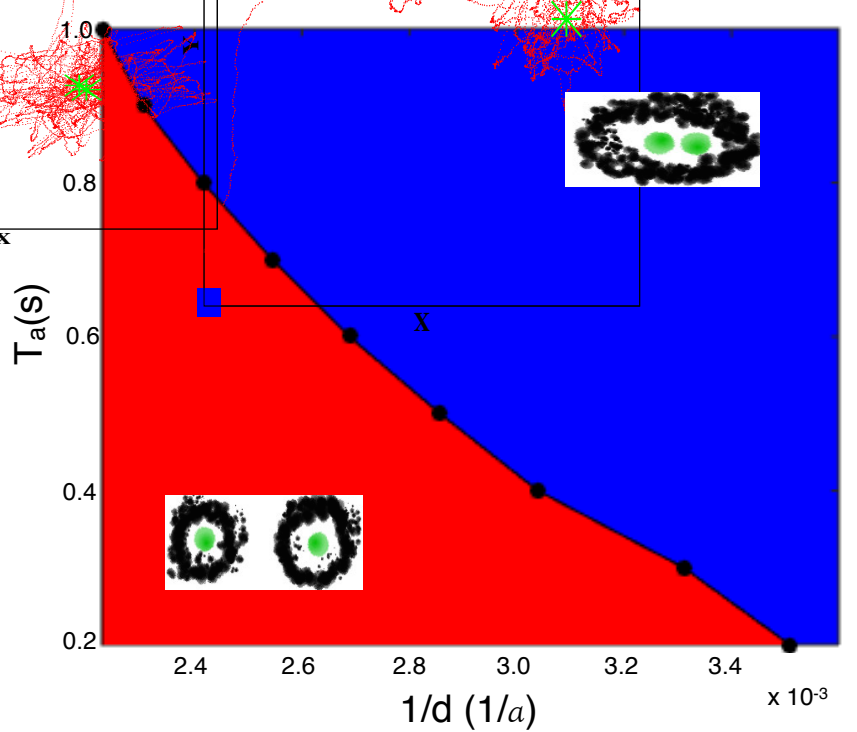

